\title{
Carbon Nanotubes-Based Electrochemical Sensing for Cell Culture Monitoring
}

\author{
Cristina Boero*, Sandro Carrara*, Giovanna Del Vecchio ${ }^{\dagger}$, Giuseppe D. Albini ${ }^{\ddagger}$, Laura Calzà ${ }^{\dagger}$ and Giovanni De Micheli* \\ * Ecole Polytechnique Fédérale de Lausanne - CH - 1015 Lausanne \\ $\dagger$ Universitá di Bologna - IT - 40064 Ozzano Emilia(BO) \\ ‡ - IT - 27053 Lungavilla (PV) \\ Email: cristina.boero@epfl.ch
}

\begin{abstract}
Monitoring of metabolic compounds, such as glucose and lactate, is extensively reported in literature, especially for clinical purposes. Instead, the application of such technologies for monitoring metabolites in cell cultures has not been explored. From one side, such devices can provide information to the current state-of-the-art of cell lines, particularly those which are not fully known, as stem and embryonic cells. On the other hand, those systems can pave the way to fully automation for growing cell cultures, when coupled with robots for feeding.

Among different presented strategies to develop biosensors, carbon nanotubes exhibit great properties, particularly suitable for biosensing. In this work nanostructured electrodes by using multi-walled carbon nanotubes are presented for the detection of glucose and lactate. Firstly, some results from simulations are illustrated in order to foresee the behavior of carbon nanotubes depending on their orientation, when they are dispersed onto the electrode surface. Then, such developed biosensors are characterized in terms of sensitivity and detection limit, and are compared to previously published results. Finally, monitoring of a cell culture is performed and the behavior of metabolites is analyzed as biosensors validation.
\end{abstract}

\section{INTRODUCTION}

Glucose and lactate biosensors have been largely reported in literature for clinical purposes. On the other hand, metabolites biosensors applied to cell cultures have not been widely studied in the past researches. Glucose detection is interesting from the point-of-view to monitor its uptake by the cells, in order to deeply understand metabolic mechanisms. Another metabolite of interest is lactate, which is related to anaerobic metabolism due to hypoxia conditions.

One possible strategy to develop biosensors for the aforementioned compounds is to employ amperometric enzyme-based biosensors. For both the metabolites, electrodes have been structured with polymeric matrices, sol-gel, cross-linker, and mediators [1] in order to optimize sensor response. Recently, nano-materials has been considered as possible electrical connectors to directly link the redox site to the electrode surface, because of the similar dimensions of nanoparticles and redox proteins. Various nano-materials have been studied since the last ten years, including nanoparticles, nanowires and nanotubes [2].

Carbon nanotubes (CNTs) have aroused increasing interest for their electrical [3] and electrochemical properties [4] with special focus on biosensors applications [5]. Due to the small diameter of these nano-materials, from 1 up to $100 \mathrm{~nm}$, quantum-effects dominate, and therefore these nanomaterials are efficiently coupled with proteins [6]. Studies have demonstrated that CNTs promote the electron-transfer between the enzyme redox active site and the transducing electrode.[7] About the applications of such biosensors to cell cultures, to date the effort in this sense was concentrated to monitor process variables, like $\mathrm{pH}, \mathrm{pO}_{2}$, electronic impedance and temperature in bioreactors [8]. Recently, Pemberton et al. [9] developed an amperometric biosensor to monitor glucose in a liver cell line. The authors report concentration values for different densities of seeded cells after 24 hours of culture. Therefore, it is possible to envisage new emerging application areas for metabolites biosensors in cells monitoring. These biosensors can be instrumental in investigating unknown, or non well-known cell lines, as stem or embryonic cells, also during proliferation or switching state, adding important information to the state-of-the-art of the cell family. Alternatively, such biosensors can be used to monitor cell cultures and to develop automated systems to manage cell cultures, saving time to human work.

The objective of the present work is the development of nanostructured electrodes by using Multi-Walled Carbon Nanotubes (MWCNT) for the detection of glucose and lactate. Results from simulations regarding CNTs behavior are presented to demonstrate why this type of nano-materials is suitable for biosensing. A comparison between non-nanostructured and nanostructured electrodes is investigated to demonstrate that detection is not possible without carbon nanotubes. Then, a fully characterization of the developed biosensors is reported, and performance of the proposed biosensors are compared with literature. Finally, nanostructured electrodes are functionalized with the enzyme probe and employed in a cell culture to monitor glucose uptake and lactate production. For this study the SN56 cell line is used as a suitable model to study the effects of hypoxia on brain cells.

\section{MATERIALS AND METHODS}

\section{A. Chemicals}

Carbon paste screen-printed electrodes (SPE - model DRP110) and multi-walled carbon nanotubes were purchased from Dropsens (Spain). The electrodes are made of a graphite 
working electrode, which presents an active area equal to $13 \mathrm{~mm}^{2}$, a counter electrode, also made of graphite, and a reference electrode, which is made of $\mathrm{Ag} / \mathrm{AgCl}$. The total area of the cell is $22 \mathrm{~mm}^{2}$. Multi-walled carbon nanotubes (diameter $10 \mathrm{~nm}$, length 1-2 $\mu \mathrm{m}$ ) were purchased in powder (90\% purity), and subsequently diluted in chloroform to the concentration of $1 \mathrm{mg} \mathrm{ml}^{-1}$ [2]. Samples were then sonicated in order to obtain an homogeneous solution.

Glucose oxidase from Aspergillus Niger (GOD, EC 1.1.3.4, 129.9 units/mg solid), lactate oxidase from Pediococcus species (LOD, EC 1.13.12.4, $\geq 20$ units/mg solid), D-(+)glucose, and lithium L-lactate were purchased from SigmaAldrich (Switzerland) in lyophilized powder. All the proteins were dissolved in Phosphate Buffer Saline (PBS) 0.01 M at pH 7.4, while glucose and lactate were dissolved in Milli-Q.

\section{B. Cell cultures}

SN56 cell line (clone SN56.B5.G4), derived from the fusion of septal neurons of postnatal day 21 mice with N18TG2 murine neuroblastoma cells [10], was a generous gift from Prof. Wainer (Emory University, Atlanta, GA, USA). Proliferating cells were maintained in Dulbecco's modified Eagle's medium (DMEM, from Sigma-Aldrich, Switzerland), supplemented with $10 \%$ fetal bovine serum (FBS, Gibco/BRL, Rockville, MD, USA), $2 \mathrm{mM}$ l-glutamine (from SigmaAldrich), and $40 \mathrm{U} \mathrm{ml}^{-1}$ penicillin/streptomycin (from Gibco), in $25 \mathrm{~cm}^{2}$ culture flasks (Corning, New York, NY, USA) in a $5 \% \mathrm{CO}_{2}$ atmosphere at $37^{\circ} \mathrm{C}$. Medium in the stock flasks was changed every 48 hours and the cells were sub-cultured when they reached $80-90 \%$ of confluence.

For experiments, cells were seeded at $5 \times 10^{3}, 25 \times 10^{3}$ and $203 \times 10^{3}$ cells $\mathrm{cm}^{-2}$, after four passages from thawing. Then, cells were cultured onto un-coated wells in proliferating conditions for 48 hours. The surnatant medium was collected from the flasks at different cell densities after 0, 4, 24 and 48 hours after seeding. Each sample was then diluted 1:10 in PBS for electrochemical measurements. Then, samples were freezed, and thawed when measurements were performed.

\section{Preparation of electrodes}

Nanostructured SPEs were prepared by using MWCNT and the probe enzymes. To prepare the MWCNT modified SPE, $40 \mu \mathrm{l}$ of the MWCNT-chloroform solution wad deposited by drop casting ( $5 \mu \mathrm{l}$ each time) onto the working electrode and it was allowed to dry. Then, $20 \mu \mathrm{l}$ of glucose or lactate oxidase $\left(15 \mathrm{mg} \mathrm{ml}^{-1}\right.$ and $125 \mathrm{mg} \mathrm{ml}^{-1}$, respectively) were dropped onto the working electrode and stored overnight at $+4^{\circ} \mathrm{C}$, in order to allow the adsorption of the proteins onto the electrode surface. Then, the drop was rinsed out with Milli-Q and the electrode was conditioned for 10 minutes at constant potential $(+550 \mathrm{mV})$ before the first use. All the functionalized electrodes were stored at $+4^{\circ} \mathrm{C}$ and covered with PBS, when not used.

\section{Apparatus}

The electrochemical response of electrodes is investigated by chronoamperometries under aerobic conditions. Electro- chemical measurements were acquired by using Versastat 3 potentiostat (Princeton Applied Technologies). For calibration and investigation of the detection limit, the electrode was dipped into the PBS solution with a volume of $25 \mathrm{ml}$ under stirring conditions. A volume of $25 \mu \mathrm{l}$ per step of the target molecule was successively added into the solution with a timestep of 2 minutes. In the case of measurements of DMEM, the electrodes were covered with a drop of $100 \mu \mathrm{l}$ of the diluted medium. In both the cases, the applied potential was $+550 \mathrm{mV}$ vs $\mathrm{Ag} / \mathrm{AgCl}$.

\section{E. Theory of carbon nanotube emissions}

It is already known that carbon nanotubes have considerable electron field emission properties. They are used to enhance the emissivity of electrodes made of various materials [11]. CNTs are also used for enhancing sensitivity of biosensors [2], [11]. Many theoretical works have been published related to the field emission at the tip of individual capped CNTs, based both on the density functional theory or the Green's function theory [12], [13], or even using the symmetry properties of CNTs for solving the Schroedinger equation [14], [15]. On the other hand, experimental works have been made to investigate the sidewall field emission properties of CNTs aligned in bundle [16], [17]. Two conclusions were drawn in these works: the sidewall field emission obeys the Fowler-Nordheim equation ([16], [17]), and field emission performance increases with decreasing bundle diameter [17]. This property suggests that the best emission performance is from the body (the sidewall) of individual CNTs. Our aim is to derive an equation, which governs the full emission of an individual CNT including electrons emission from both sidewall and tip. The general equation needs to consider the arbitrarily oriented carbon nanotubes with respect to the field, while using a simple model to investigate the nanostructure of the electrodes. Toward this goal, we investigate the situation in which there is a CNT with the axis parallel to the substrate and, therefore, perpendicular to field $E$. The developed model is based on the following three assumptions:

1) the electron emission occurs through the CNT half surface facing the anode;

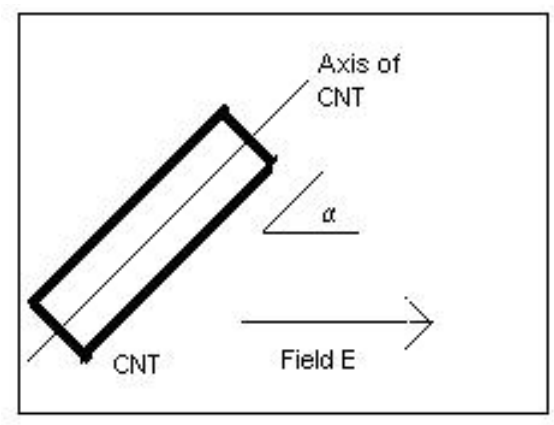

Fig. 1. Model for a linear segment of a CNT. 


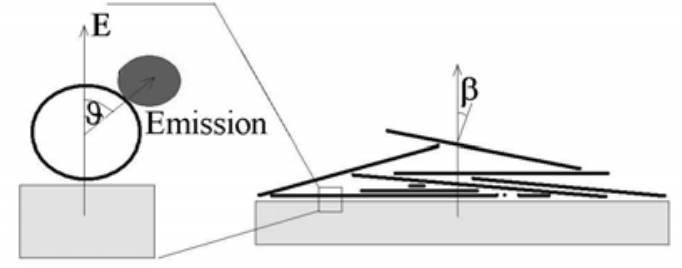

Lateral View

Fig. 2. Carbon nanotubes randomly organized onto the electrode surface.

2) the field $E$ is uniform both in direction and intensity in the neighborhood of the substrate and of the CNT;

3 ) the current emitted across the surface $\sigma$ obeys the Fowler-Nordheim equation considering the projection of $E$ on the normal to $\sigma$. In other words, assuming $\sigma$ as a flat surface, the Fowler-Nordheim equation is

$$
I=K_{1} \sigma E_{\perp}^{2} \exp \left(-\frac{K_{2}}{E_{\perp}}\right),
$$

where $I$ is the current emitted across $\sigma, E_{\perp}$ is the projection of $E$ on the normal to $\sigma$ and $K_{1}$ and $K_{2}$ are suitable constants.

Then, if we assume that the axis of CNT is not perpendicular to the field, but forms an angle $\alpha$ (as illustrated in Fig. 1), and we express variables in a cylindrical coordinate system, with the axis of CNT as z-axis, whose origin is at one end of $\mathrm{CNT}$, and the direction of $\mathrm{E}$ as origin of coordinate $\vartheta$, we can integrate on the portion of surface of CNT facing the anode, and obtain the total current emitted across the side surface:

$i_{S}(E, \alpha)=K_{1} \rho E^{2} L \int_{-\frac{\pi}{2}}^{\frac{\pi}{2}}(\cos \vartheta \sin \alpha)^{2} \exp \left(-\frac{K_{2}}{E \cos \vartheta \sin \alpha}\right) d \vartheta$

In this case also the emission from the CNT tip facing the anode should be considered. The current from the tip obeys the Fowler-Nordheim equation [14] which is written as

$$
i_{T}(E, \alpha)=K_{1}^{\prime} A(E \cos \alpha)^{2} \exp \left(-\frac{K_{2}^{\prime}}{E \cos \alpha}\right) .
$$

The entire current emitted by a CNT oriented according the angle $\alpha$ with respect to the field is

$$
i(E, \alpha)=i_{S}(E, \alpha)+i_{T}(E, \alpha) .
$$

In case of a water environment, such as experiments with biosensors during the detection of glucose and lactate in the cell medium, the emitted current is further enhanced by the presence of water molecules close to the nanotubes surface [12].

\section{F. Monte Carlo Simulations}

In order to investigate the role of carbon nanotubes in our structured electrodes, numerical simulations are implemented considering (4). Specifically, Monte Carlo simulations are performed to obtain the final distribution of the carbon nanotubes onto our screen-printed electrodes. In fact, it is possible to

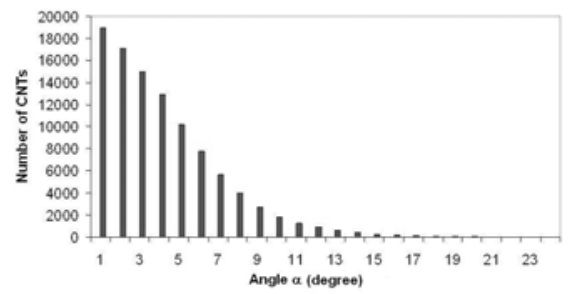

Fig. 3. Monte Carlo simulation results for the distribution of the angle between the longitudinal axis of CNTs and the electrode.

imagine that active redox species are in contact with all the available surface of a carbon nanotube drop cast onto the surface of a screen-printed electrode. Consequently, electrons emission toward redox species would happen both from the sidewall and from the tip. The electric field is typically normal to the baseline of the electrode. However, a screen-printed electrode has a mean corrugation in the $10-\mathrm{nm}$ scale. Then, the single carbon nanotube is positioned forming a certain angle with respect to the electric field, which would be typically normal to the electrode baseline, depending on the region where it was landing, as shown by Fig. 2. Therefore, Monte Carlo simulations are performed to simulate the landing of each single carbon nanotube onto the electrode surface. The final situation reached by each simulation is similar to the drawing in Fig. 2. The figure shows some carbon nanotubes differently organized onto the electrode surface, each of those presenting a different angle with respect to the electric field, which is orthogonal to the electrode surface.

\section{RESULTS AND DISCUSSION}

\section{A. Electrons emission from carbon nanotubes}

Initial results obtained from Monte Carlo simulations include the angle distribution of CNTs longitudinal axis with respect to the electrode surface (angle $\alpha$ ). Fig. 3 illustrates such a distribution for one of the simulations. The large majority of carbon nanotubes is distributed to the lower angles and the number of nanotubes with larger angle decays very rapidly for angles larger than 10 degrees. Consequently, the main contribution to the total current is due to sidewall emission, since the majority of CNTs are almost parallel to the surface. Next simulations will involve investigation of emission current from carbon nanotube tips and sidewalls in order to verify the hypothesis in equation (4).

\section{B. Improvements with nanostructured electrodes}

The improvements of nanostructured electrodes by using multi-walled carbon nanotubes were previously demonstrated in the case of hydrogen peroxide [18]. The enhancement in terms of sensitivity between the case of non-nanostructured and nanostructured electrodes is about 7 times, also suggesting a similar improvement in terms of performance for oxidasebased biosensors.

Detection of glucose and lactate in the range of $\mathrm{mM}$ are 


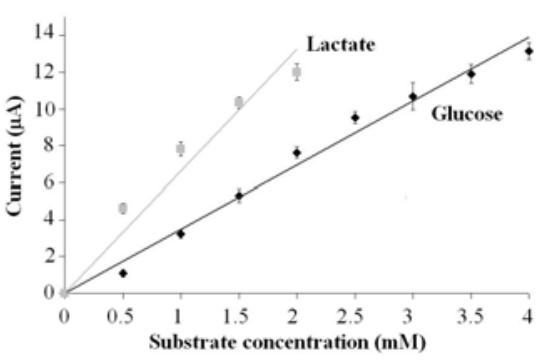

Fig. 4. Calibration lines for glucose and lactate in the concentration range of interest.

performed for electrodes without and with nanostructuration, to evaluate the performance of the two strategies. In the case of bare electrode, the detection is not possible within the range of interest for both the cases (data not shown). On the other hand, the electrodes with carbon nanotubes show good sensitivity and a current range in the order of $\mu \mathrm{A}$. These results are coherent with literature [2]. A lot of studies [1] overcame the difficulty to directly couple the enzyme with the electrode by using a mediator. The role of the mediator is shuttling electrons from the protein to the electrode surface, despite of the complexity of the system. From these results it is possible to assert that nano-materials are good candidates to replace the role of mediators, since they allow the direct electron-transfer from the active site of the enzyme to the electrode surface. Moreover, nano-materials can be grown onto the electrode surface during microfabrication, enabling integration with VLSI chips.

The range of detection is chosen considering that the concentration of glucose in DMEM is at maximum $22.4 \mathrm{mM}$ (4.5 $\mathrm{g} \mathrm{l}^{-1}$ of glucose). Since it is not possible to perform measurements in pure DMEM, due to the interferences arising from easily electro-oxidizable substances at the same oxidizing potential, all the measurements are performed in diluted DMEM (dilution 1:10 in PBS), so that the maximum concentration of interest is $2.24 \mathrm{mM}$. Moreover, linear range of the developed biosensor is not able to cover all the range of interest, so dilution is a proper solution. For the case of lactate,

TABLE I

PERFORMANCES OF NANOSTRUCTURED-BASED BIOSENSORS FOR GLUCOSE DETECTION

\begin{tabular}{|c|c|c|}
\hline Method & $\begin{array}{c}\text { Sensitivity } \\
\left(\mu \mathrm{mM}^{-1} \mathrm{~cm}^{-2}\right)\end{array}$ & $\begin{array}{c}\text { Linear range } \\
(\mathrm{mM})\end{array}$ \\
\hline \hline MWCNT - Nafion $®[19]$ & 171.2 & $0.005-0.5$ \\
\hline MWCNT - PtNP - CS/MTOS [20] & 69.9 & $0.012-6$ \\
\hline MWCNT - Nafion $®[21]$ & 4.7 & $0.025-2$ \\
\hline Present work & 27.7 & $0.055-4$ \\
\hline
\end{tabular}

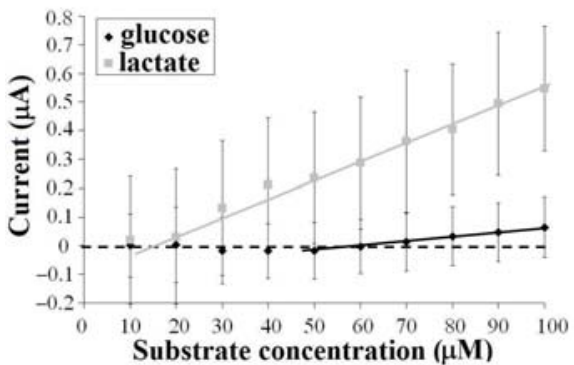

Fig. 5. Detection limit for glucose and lactate biosensors.

Hwang et al. [22] reported a value of $10 \mathrm{mM}$ in the case of murine embryonic stem cells. The range from 0.5 to $4 \mathrm{mM}$ for glucose and from 0.5 to $2 \mathrm{mM}$ for lactate are considered as the most suitable for the objective of the present research, by considering the dilution of the DMEM.

\section{Calibration}

Calibration lines are worked out from chronoamperometries within the concentration range of interest (from 0.5 to $4 \mathrm{mM}$ for glucose and from 0.5 to $2 \mathrm{mM}$ for lactate, see Fig. 4). Both for glucose and lactate, substrates were dissolved in Milli-Q and added into the PBS solution every $120 \mathrm{~s}$, to allow the system to reach the steady-state. The response time of the system after each addition is around $30 \mathrm{~s}$ (data not shown). The calibration curve in the case of glucose detection shows a sensitivity of $27.7 \mu \mathrm{A} \mathrm{mM}{ }^{-1} \mathrm{~cm}^{-2}$, while in the case of lactate detection the sensitivity is of $40.1 \mu \mathrm{A} \mathrm{mM}^{-1} \mathrm{~cm}^{-2}$. The detection limit is also investigated for both the substrates, to determine the lowest limit of the linear range. As shown in Fig. 5, detection limit for glucose biosensor is $55 \mu \mathrm{M}$, while it is $15 \mu \mathrm{M}$ for lactate. It is possible to compare the obtained sensitivities and detection limit with what was found in literature for similar nanostructured biosensors. Table I lists previous works regarding glucose detection. The current research obtained a sensitivity in the same order of magnitude with respect to what was obtained in literature, except for the case of Rahman et al. [19], where the value was obtained for a narrower detection range (from 1 to $500 \mu \mathrm{M}$ ), not useful for the purpose of the present work. Kang et al. [20] obtained

TABLE II

PERFormanCES OF NANOSTRUCTURED-BASED BIOSENSORS FOR LACTATE DETECTION

\begin{tabular}{|c|c|c|}
\hline Method & $\begin{array}{c}\text { Sensitivity } \\
\left(\mu \mathrm{A} \mathrm{m}^{-1} \mathrm{~cm}^{-2}\right)\end{array}$ & $\begin{array}{c}\text { Linear range } \\
(\mathrm{mM})\end{array}$ \\
\hline \hline LDH - MWCNT - CS [23] & 8.3 & $0.005-1.2$ \\
\hline MWCNT - PVI - CS [24] & 19.7 & $0.005-1$ \\
\hline MWCNT - sol-gel [25] & 2.097 & $0.3-2$ \\
\hline Present work & 40.1 & $0.015-2$ \\
\hline
\end{tabular}




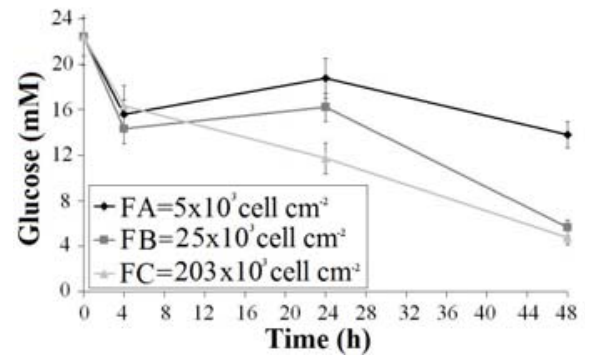

Fig. 6. Glucose uptake in cell line SN56 after 4, 24, and 48 hours.

a higher value of sensitivity, but it is important to consider that they employed a sol-gel matrix combined with carbon nanotubes and nanoparticles, which can enhance the efficiency, despite of the cost and complexity of the sensor.

Regarding lactate detection, from Table II note that the obtained sensitivity is twice the highest value reported in literature, as another indication that nanomaterials, such as carbon nanotubes, functionalized with oxidases are greatest substrate for biosensors. Moreover, in the case of lactate it is possible to assert that a further structuration of the electrode with polymers, as in the case of Cui et al. [24] does not exhibit better performance in terms of detection.

These results also validate the phenomenon reported from Carrara et al. [6] regarding the inverse linear correlation between sensitivity and detection limit when using nanomaterials for biosensing. Comparing the obtained values in this work with non-nanostructured biosensors ([26], [27]), an improvement of performance with respect to the case of mediated electron-transfer is demonstrated. The advantages brought from nanomaterials are really evident, especially in ranges of concentration which are of interest for a large pool of applications, and they make carbon nanotubes the key-element for the future of nano-biosensing.

\section{Detection of metabolites in cell cultures}

As mentioned above, DMEM is diluted with PBS in a rate of 1 to 10 , to be in the linear range of biosensors. Measurements are collected at the time of the seeding, and 4, 24 and 48 hours later. The same diluted medium is used for glucose and lactate detection: the probe enzyme onto the electrode is different, but no cross talk or interferences are noticed from the other substrate during measurements. The behavior of glucose uptake and lactate production is depicted in Fig. 6 and in Fig. 7 along 48 hours of cell culture for different densities of cells. Note that glucose uptake and lactate production are different for various cell densities. It means that with the developed biosensors is possible to recognize different cell densities and behaviors are clearly distinguishable. Another important point is that glucose uptake and lactate production shapes are quite symmetric. The lack of feeding, i.e. glucose, induces the production of lactate, which is a mark of cell suffering. Consequently, the symmetry of trends is a further confirmation of this phenomena. On the other hand, in the lowest cell

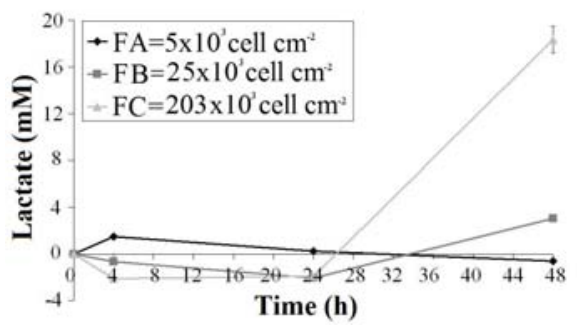

Fig. 7. Lactate production in cell line SN56 after 4, 24, and 48 hours.

density the developed biosensor recorded an uptake of glucose in the first 4 hours, but afterwards the cells have enough feeding to survive more than 48 hours without changing the medium. It is also confirmed in two ways by the fact that lactate production is almost zero. On the contrary, for the highest cell density, glucose uptake increases along time, while lactate production reached the maximum after 48 hours. Cells are still living, but the change of medium is required. From this perspective, the developed biosensors can be a useful instrument to investigate mechanisms, which occur during cell proliferation, while simultaneously provide information to the operators about cell culture conditions and right timing for further cell feeding.

Moreover, observation of changes in the cell culture leads to develop skillful technologies for unknown cell systems. Biosensors developed in this study were tested and validated in a standard cell line, but such emerging devices can be applied to more complex cell lines, like stem cells, where there is a lack of knowledge about the mechanisms that unfold when cells switch from the stem state to the differentiate state.

It is necessary to improve the developed biosensor in order to avoid the recording of values of current which corresponds to negative lactate concentration. A possible explanation for this phenomena could be the fact that electrochemical measurements are strongly affected by $\mathrm{pH}$. Medium $\mathrm{pH}$ has to be strictly controlled and it can change along cell line development, due to metabolites and products secreted by cells during their growth. For this reason, the next step will be the integration and correction of concentration values according to the variation of $\mathrm{pH}$ solution, in order to achieve more accurate measurements.

\section{CONCLUSION}

This work presents the development of amperometric enzyme-based biosensors nanostructured by using multiwalled carbon nanotubes for metabolites detection in cell culture medium. In particular, biosensors are functionalized for the detection of glucose and lactate by means of oxidases. Monte Carlo simulations are used to model the distribution of CNTs onto the electrode surface. Results show that the majority of carbon nanotubes forms low angles with the substrate. Consequently, it could be inferred that sidewalls emission is more significant compared to tips emission.

Electrochemical measurements are performed for both 
metabolites by means of chronoamperometries. Sensitivity of $27.7 \mu \mathrm{A} \mathrm{mM}^{-1} \mathrm{~cm}^{-2}$ is reached in the case of glucose, while a value of $40.1 \mu \mathrm{A} \mathrm{mM} \mathrm{m}^{-1} \mathrm{~cm}^{-2}$ is obtained in the case of lactate detection. Detection limit is also investigated in terms of the smallest detectable concentration: a value of $55 \mu \mathrm{M}$ is obtained for glucose, while the detection limit for lactate is $15 \mu \mathrm{M}$. From a comparison with nanostructured biosensors by using carbon nanotubes in literature, the presented results are in the same order of magnitude of what was previously found for glucose, while for lactate the highest sensitivity is obtained in this work. These results are a further confirmation of how carbon nanotubes can improve the performance of biosensors. Once calibration is performed, detection of both these metabolites is performed in cell medium for three different cell densities. Measurements are exploited with nanostructured electrodes functionalized with one of the two oxidases with diluted medium. Two behaviors are obtained, one for glucose uptake and one for lactate production. Glucose concentration decreases along time, more in the case of higher cell density, while lactate production increases. Such results illustrate the possibility to monitor metabolites in cell cultures by means of amperometric biosensors, enabling to develop fully integrated Petri dishes able to detect metabolites, targeting on automated system. On the other hand, developed biosensors can add useful information to the knowledge of line, such embryonic or stem cell, which are not completely well-known.

\section{ACKNOWLEDGMENT}

The authors would like to thank CIME facility, especially Dr. M. Cantoni and Ms. F. Bobard to acquire SEM images, and Dr. V. Pavlidis for help in manuscript revision. The research is financed by SNF Sino-Swiss cooperation project n. IZLCZ2 123967 and by EPFL - Integrated Center SI.

\section{REFERENCES}

[1] R. Garjonyte, Y. Yigzaw, R. Meskys, A. Malinauskas, and L. Gorton Prussian blue- and lactate oxidase-based amperometric biosensor for lactic acid. Sensors and Actuators B: Chemical, 79(1):33 - 38, 2001.

[2] S. Carrara, V.V. Shumyantseva, A.I. Archakov, and B. Samorì. Screenprinted electrodes based on carbon nanotubes and cytochrome p450scc for highly sensitive cholesterol biosensors. Biosensors and Bioelectronics, 24(1):148 - 150, 2008.

[3] V. Bavastrello, S. Carrara, M.K. Ram, and C. Nicolini. Optical and electrochemical properties of poly(o-toluidine) multiwalled carbon nanotubes composite langmuirschaefer films. Langmuir, 20(3):969-973, 2004.

[4] V. Bavastrello, E. Stura, S. Carrara, V. Erokhin, and C. Nicolini. Poly(2,5-dimethylaniline)-mwnts nanocomposite: a new material for conductometric acid vapours sensor. Sensors and Actuators B: Chemical, 98(2-3):247 - 253, 2004.

[5] S. Carrara, V. Bavastrello, D. Ricci, E. Stura, and C. Nicolini. Improved nanocomposite materials for biosensor applications investigated by electrochemical impedance spectroscopy. Sensors and Actuators B: Chemical, 109(2):221 - 226, 2005.

[6] S. Carrara, C. Boero, and G. De Micheli. Quantum dots and wires to improve enzymes-based electrochemical bio-sensing. Proceedings of the 4th International Conference Nano-Nets, Luzern, Switzerland, October 2009, LNICTS 20 - Springer, Berlin 2009:189-199, 2009.

[7] C. Cai and J. Chen. Direct electron transfer of glucose oxidase promoted by carbon nanotubes. Analytical Biochemistry, 332:75-83, 2004.
[8] J. Wiest, M. Schmidhuber, J. Ressler, A. Scholz, M. Brischwein, and B. Wolf. Cell based assays for diagnostic and therapy on multiparametric biosensor chips with an intelligent mobile lab. IFMBE Proc., 10:132135, 2005.

[9] R.M. Pemberton, J. Xu, R. Pittson, N. Biddle, G.A. Drago, S.K. Jackson, and J.P. Hart. Application of screen-printed microband biosensors to end-point measurements of glucose and cell numbers in hepg2 cell culture. Analytical Biochemistry, 385(2):334 - 341, 2009.

[10] H.J. Lee, D.N. Hammond, T.H. Large, and B.H. Wainer. Immortalized young adult neurons from the septal region: generation and characterization. Developmental Brain Research, 52(1-2):219 - 228, 1990.

[11] J.J. Gooding. Nanostructuring electrodes with carbon nanotubes: A review on electrochemistry and applications for sensing. Electrochimica Acta, 50(15):3049 - 3060, 2005.

[12] L Qiao, W T Zheng, Q B Wen, and Q Jiang. First-principles densityfunctional investigation of the effect of water on the field emission of carbon nanotubes. Nanotechnology, 18(15):155707 (6pp), 2007.

[13] H. Roohi and S. Bagheri. Atomic and electronic structures of finite single-walled bn nanotubes: Hybrid dft calculations. Journal of Molecular Structure: THEOCHEM, 856(1-3):46 - 58, 2008.

[14] A. Mayer, N. M. Miskovsky, and P. H. Cutler. Theoretical comparison between field emission from single-wall and multi-wall carbon nanotubes. Phys. Rev. B, 65(15):155420, 2002.

[15] A. Mayer and J.-P. Vigneron. Real-space formulation of the quantummechanical elastic diffusion under $n$-fold axially symmetric forces. Phys. Rev. B, 56(19):12599-12607, 1997.

[16] Y. Chen, D.T. Shaw, and L. Guo. Field emission of different oriented carbon nanotubes. Applied Physics Letters, 76(17):2469-2471, 2000.

[17] D. McClain, M. DeRoss, N. Tavan, J. Jiao, C.M. McCarter, R.F. Richards, S.Mesarovic, C.D. Richards, and D.F. Bahr. Effect of diameter on electron field emission of carbon nanotube bundles. In Mater. Res. Soc. Symp. Proc., volume 901E, 2006.

[18] C. Boero, S. Carrara, and G. De Micheli. Sensitivity enhancement by carbon nanotubes: Applications to stem cell cultures monitoring. In Research in Microelectronics and Electronics, 2009. PRIME 2009. Ph.D., pages 72-75, July 2009.

[19] M.M. Rahman, A. Umar, and K. Sawada. Development of amperometric glucose biosensor based on glucose oxidase co-immobilized with multiwalled carbon nanotubes at low potential. Sensors and Actuators B: Chemical, 137(1):327 - 333, 2009.

[20] X. Kang, Z. Mai, X. Zou, P. Cai, and J. Mo. Glucose biosensors based on platinum nanoparticles-deposited carbon nanotubes in sol-gel chitosan/silica hybrid. Talanta, 74(4):879 - 886, 2008.

[21] Y.C. Tsai, S.C. Li, and J.M. Chen. Cast thin film biosensor design based on a nafion backbone, a multiwalled carbon nanotube conduit, and a glucose oxidase function. Langmuir, 21(8):3653-3658, April 2005.

[22] Y.S. Hwang, J. Cho, F. Tay, J.Y.Y. Heng, R.Ho, S.G. Kazarian, D.R. Williams, A.R. Boccaccini, J.M. Polak, and A.Mantalaris. The use of murine embryonic stem cells, alginate encapsulation, and rotary microgravity bioreactor in bone tissue engineering. Biomaterials, 30(4):499 $-507,2009$.

[23] Y.C. Tsai, S.Y. Chen, and H.W. Liaw. Immobilization of lactate dehydrogenase within multiwalled carbon nanotube-chitosan nanocomposite for application to lactate biosensors. Sensors and Actuators B: Chemical, 125(2):474 - 481, 2007.

[24] X. Cui, C. M. Li, J. Zang, and S. Yu. Highly sensitive lactate biosensor by engineering chitosan/pvi-os/cnt/lod network nanocomposite. Biosensors and Bioelectronics, 22(12):3288 - 3292, 2007.

[25] J. Huang, Z. Song, J. Li, Y. Yang, H. Shi, B. Wu, J. Anzai, T. Osa and Q. Chen. A highly-sensitive l-lactate biosensor based on sol-gel film combined with multi-walled carbon nanotubes (mwents) modified electrode. Materials Science and Engineering: C, 27(1):29 - 34, 2007.

[26] Y. Himuro, M. Takai, and K. Ishihara. Poly(vinylferrocene-co-2hydroxyethyl methacrylate) mediator as immobilized enzyme membrane for the fabrication of amperometric glucose sensor. Sensors and Actuators B: Chemical, 136(1):122 - 127, 2009.

[27] R.M. Pemberton, R. Pittson, N. Biddle, and J.P. Hart. Fabrication of microband glucose biosensors using a screen-printing water-based carbon ink and their application in serum analysis. Biosensors and Bioelectronics, 24(5):1246 - 1252, 2009. Selected Papers from the Tenth World Congress on Biosensors Shangai, China, May 14-16, 2008. 